\title{
Spatio-Temporal Analysis of Throughput for Single-Hop CSMA Networks
}

\author{
Mehmet Koseoglu and Ezhan Karasan, Member, IEEE
}

\begin{abstract}
Throughput model for non-persistent CSMA networks which was proposed by Kleinrock and Tobagi has been widely used, although it provides a loose lower bound when nodes are distributed in a large area because the analysis assumes that the propagation delay between each pair of users equals to the largest propagation delay in the network. We present a throughput analysis which considers the spatial distribution of nodes. We obtain a simple throughput expression which predicts throughput with an $8 \%$ maximum error whereas the earlier model results in a $44 \%$ error when the maximum propagation delay equals to the packet transmission time.
\end{abstract}

Index Terms-Spatio-temporal analysis, single-hop CSMA.

\section{INTRODUCTION}

A CSMA network with non-negligible propagation delay is sensitive to spatio-temporal effects because the locations of the nodes affect their time of exposure to the transmissions of other nodes in the network. In the seminal analysis of nonpersistent CSMA [1], these effects are ignored by assuming that the propagation delay between each node in the network equals to the largest propagation delay in the network. This assumption results in a loose lower bound for the network throughput [2]. We here propose a novel analysis of nonpersistent CSMA without ignoring the spatial distribution of the nodes, which predicts the throughput much more accurately.

The model proposed in [1] is still frequently referred in the studies on the performance analysis of terrestrial networks [3][7] and on the performance analysis of underwater networks where the propagation delay is much higher than the terrestrial networks [8]. Despite the model's widespread usage, it is not accurate for scenarios where the nodes are distributed over a large area. We propose an exact model of non-persistent CSMA and, then, an approximate simpler model which results in an $8 \%$ estimation error whereas the model in [1] results in a $44 \%$ error when the maximum propagation delay in the network equals to the packet transmission time.

The rest of the letter is organized as follows: The next section describes the system model that we study, we explain the main idea behind our analysis in Sec. III. Derivation of the throughput expression is presented in Sec. IV and numerical results are given in Sec. V.

\section{SCENARIO DESCRIPTION}

We consider uniformly distributed nodes over a circular area which are transmitting to the base station located at the

Manuscript received October 11, 2013. The associate editor coordinating the review of this letter and approving it for publication was P. Serrano.

The authors are with the Department of Electrical and Electronics Engineering, Bilkent University, TR-06800, Bilkent, Ankara, Turkey (e-mail: $\{$ kmehmet, ezhan\}@ee.bilkent.edu.tr).

Digital Object Identifier 10.1109/LCOMM.2014.020414.132292 center. We assume that a large number of nodes are spatially distributed according to a homogeneous Poisson point process. We only consider the traffic from the nodes to the base station, and we assume that the nodes always have a packet to send. We do not consider retransmissions; that is, each node has always a single packet to send. Nodes attempt to transmit according to the Poisson distribution where $\lambda$ denotes the total attempt rate in the network. We also assume that consecutive transmissions are independent, i.e., we analyze a transmission in isolation from a previous transmission.

The largest propagation delay normalized to the packet transmission time is denoted as $T$; hence a node can have a maximum normalized propagation delay of $T / 2$ to the base station. A node $n_{i}$ is described by its polar coordinates $\left(r_{i}, \theta_{i}\right)$ where $r_{i} \leq \frac{T}{2}$ is the propagation delay between $n_{i}$ and the base station, such that all distances are expressed in terms of normalized propagation delay.

\section{MAIN IDEA}

The main difference between the proposed analysis of CSMA and the traditional analysis of CSMA [1] is that we consider the spatial distribution of nodes in our analysis. Due to the spatial distribution of nodes, some nodes may stay exposed to a finished transmission for a longer time than other nodes or may be exposed to a starting transmission earlier than other nodes. This results in a time-varying packet arrival rate at the base station different than the time-homogeneous arrival rate assumption used in [1]. In this part, we explain this phenomenon.

\section{A. Decreasing arrival rate after the start of a transmission}

In the CSMA protocol, at the beginning of a transmission, the transmission undergoes a vulnerable period of $T$ during which a colliding transmission may arrive. In the traditional analyses of CSMA, it is assumed that colliding transmissions arrive at a fixed rate during this vulnerable period. However, in practice, as time progresses within the vulnerable period, the arrival rate of colliding transmissions reduces as more nodes are exposed to the started transmission.

A sample network where node $n_{0}$ has started a transmission is depicted in Fig. 1 and the timeline of the transmission is depicted in Fig. 2. Assume $n_{0}$ has started a transmission at $t=t_{0}$. Node $n_{1}$ becomes exposed to this transmission at $t=t_{0}+d\left(n_{0}, n_{1}\right)$, where $d\left(n_{0}, n_{1}\right)$ is the temporal distance between nodes $n_{0}$ and $n_{1}$. After $t=t_{0}+d\left(n_{0}, n_{1}\right)$, node $n_{1}$ cannot start a transmission since it now senses the channel busy. A transmission which is started by $n_{1}$ just before it becomes exposed, will arrive to the base station at time $t=$ $t_{0}+d\left(n_{0}, n_{1}\right)+r_{1}$. Since the transmission of $n_{0}$ will arrive 


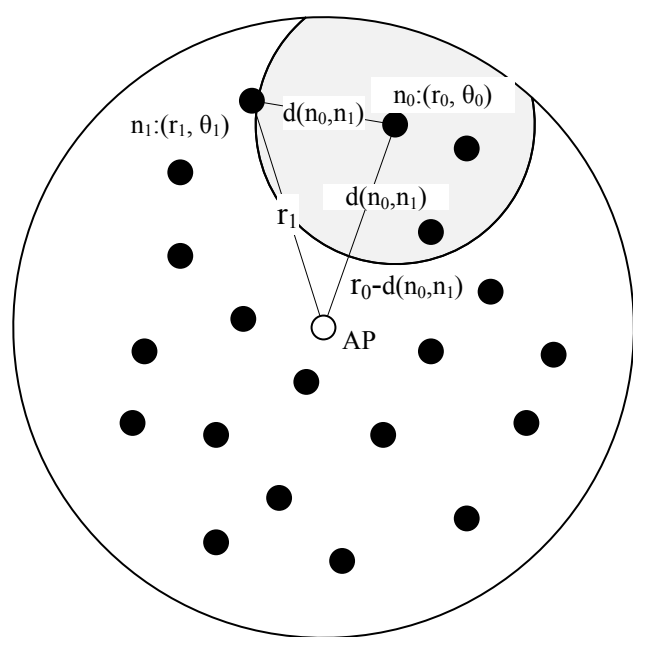

Fig. 1. A sample single-hop network scenario with marked distances. Grayed circle denotes the exposure region for the transmission by node $n_{0}$.

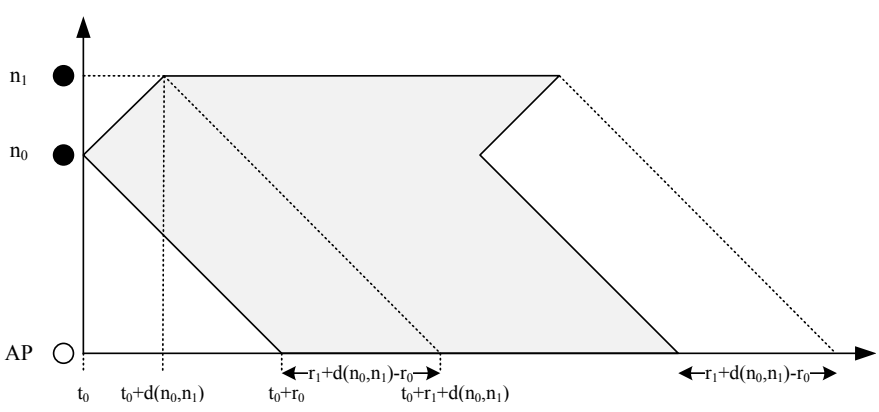

Fig. 2. Timeline for a transmission by $n_{0}$. Time difference between the arrival of $n_{0}$ 's transmission at the base station and the arrival of the latest possible transmission from $n_{1}$ is $r_{1}+d\left(n_{0}, n_{1}\right)-r_{0}$. Similarly, transmission of $n_{1}$ cannot arrive at the base station for a duration of $r_{1}+d\left(n_{0}, n_{1}\right)-r_{0}$ after the transmission of $n_{0}$ is fully received at the base station.

to the base station at time $t=t_{0}+r_{0}, n_{0}$ 's transmission stays vulnerable to $n_{1}$ 's transmission for a duration of $\left(r_{1}+\right.$ $\left.d\left(n_{0}, n_{1}\right)-r_{0}\right)$ at the beginning.

We define $\lambda_{n\left(r_{1}, \theta_{1}\right) \rightarrow n_{0}}^{s t}(t)$ as the rate of colliding transmissions from nodes located at $\left(r_{1}, \theta_{1}\right)$ to the transmission of $n_{0}$ where $t$ denotes the time passed after the transmission of $n_{0}$ arrives to the base station. The superscript $s t$ is an abbreviation of start indicating the arrival rate after the transmission starts at the base station. Since nodes are uniformly distributed within the circular region, the attempt rate of nodes located at $\left(r_{1}, \theta_{1}\right)$ is $\frac{\lambda}{\pi T^{2} / 4} \mathrm{~d} A$ where $\mathrm{d} A=r_{1} \mathrm{~d} r_{1} \mathrm{~d} \theta_{1}$. The latest time that $n_{0}$ 's transmission can experience a collision from a node located at $\left(r_{1}, \theta_{1}\right)$ is $\left(r_{1}+d\left(n_{0}, n_{1}\right)-r_{0}\right)$, so, the rate of colliding arrivals can be written as:

$$
\begin{aligned}
& \lambda_{n\left(r_{1}, \theta_{1}\right) \rightarrow n_{0}}^{s t}(t) \\
& \quad= \begin{cases}\frac{4 \lambda}{\pi T^{2}} r_{1} \mathrm{~d} r_{1} \mathrm{~d} \theta_{1} & \text { if } t<\left(r_{1}+d\left(n_{0}, n_{1}\right)-r_{0}\right) \\
0 & \text { if } t>\left(r_{1}+d\left(n_{0}, n_{1}\right)-r_{0}\right)\end{cases}
\end{aligned}
$$

where

$$
d\left(n_{0}, n_{1}\right)=\sqrt{r_{0}^{2}+r_{1}^{2}-2 r_{0} r_{1} \cos \left(\theta_{0}-\theta_{1}\right)} .
$$

Total rate of colliding transmissions from all nodes after a transmission by node $n_{0}$ is obtained by integrating over the entire circle:

$$
\lambda_{\cup_{i} n\left(r_{i}, \theta_{i}\right) \rightarrow n_{0}}^{s t}(t)=\int_{0}^{2 \pi} \int_{0}^{\frac{T}{2}} \lambda_{n\left(r_{1}, \theta_{1}\right) \rightarrow n_{0}}^{s t}(t) .
$$

To obtain the average arrival rate of colliding transmissions after a transmission by an arbitrary node in the network, $\lambda^{s t}(t)$, we take the average over all nodes, $n_{0}$. Since each node has an equal probability of starting a new transmission, we obtain

$$
\lambda^{s t}(t)=\int_{0}^{2 \pi} \int_{0}^{\frac{T}{2}} \frac{4}{\pi T^{2}} \lambda_{\cup_{i} n\left(r_{i}, \theta_{i}\right) \rightarrow n_{0}}^{s t}(t) r_{0} \mathrm{~d} r_{0} \mathrm{~d} \theta_{0} .
$$

\section{B. Increasing arrival rate after the end of a transmission}

A similar phenomenon occurs after the end of a transmission. A transmission from $n_{1}\left(r_{1}, \theta_{1}\right)$ cannot arrive for a duration of $\left(r_{1}+d\left(n_{0}, n_{1}\right)-r_{0}\right)$ after the transmission of $n_{0}$ is fully received at the base station as depicted in Fig. 2.

We define $\lambda_{n\left(r_{1}, \theta_{1}\right) \rightarrow n_{0}}^{\text {end }}(t)$ as the rate of arrivals from nodes located at $\left(r_{1}, \theta_{1}\right)$ after the transmission of $n_{0}$ finishes where $t$ denotes the time elapsed after transmission of $n_{0}$ finishes at the base station. This arrival rate is given by:

$$
\begin{aligned}
& \lambda_{n\left(r_{1}, \theta_{1}\right) \rightarrow n_{0}}^{\text {end }}(t) \\
& \quad= \begin{cases}0 & \text { if } t<\left(r_{1}+d\left(n_{0}, n_{1}\right)-r_{0}\right) \\
\frac{4 \lambda}{\pi T^{2}} r_{1} \mathrm{~d} r_{1} \mathrm{~d} \theta_{1} & \text { if } t>\left(r_{1}+d\left(n_{0}, n_{1}\right)-r_{0}\right)\end{cases}
\end{aligned}
$$

The arrival rate of starting transmissions from all nodes in the network is given by:

$$
\lambda_{\cup_{i} n\left(r_{i}, \theta_{i}\right) \rightarrow n_{0}}^{\text {end }}(t)=\int_{0}^{2 \pi} \int_{0}^{\frac{T}{2}} \lambda_{n\left(r_{1}, \theta_{1}\right) \rightarrow n_{0}}^{\text {end }}(t) .
$$

and the average arrival rate distribution can be found as:

$$
\lambda^{e n d}(t)=\int_{0}^{2 \pi} \int_{0}^{\frac{T}{2}} \frac{4}{\pi T^{2}} \lambda_{\cup_{i} n\left(r_{i}, \theta_{i}\right) \rightarrow n_{0}}^{e n d}(t) r_{0} \mathrm{~d} r_{0} \mathrm{~d} \theta_{0} .
$$

\section{Throughrut Computation}

\section{A. Linear approximation of the arrival rate function.}

Closed form expressions for the arrival rate functions given by (4) and (7) cannot be obtained. However, simulation results and numerical computation of these arrival rate functions demonstrate that these functions can be approximated by the following linear functions:

$$
\begin{aligned}
& \lambda^{s t}(t)= \begin{cases}\lambda \frac{T-t}{T} & \text { if } t \leq T, \\
0 & \text { if } t>T\end{cases} \\
& \lambda^{e n d}(t)= \begin{cases}\lambda \frac{t}{T} & \text { if } t \leq T, \\
\lambda & \text { if } t>T .\end{cases}
\end{aligned}
$$

Fig. 3 depicts the numerical computations along with the simulation results for a 1000-node network and the linear approximation for $\lambda=0.2$ and $\lambda=1$ at $T=0.9$. Numerical computations approximate the arrival rate functions very closely and the linear approximation provides a close estimation while allowing mathematical tractability.

Reduction in the accuracy at $\lambda=1$ is due to increased dependency between consecutive transmissions at a higher $\lambda$. 


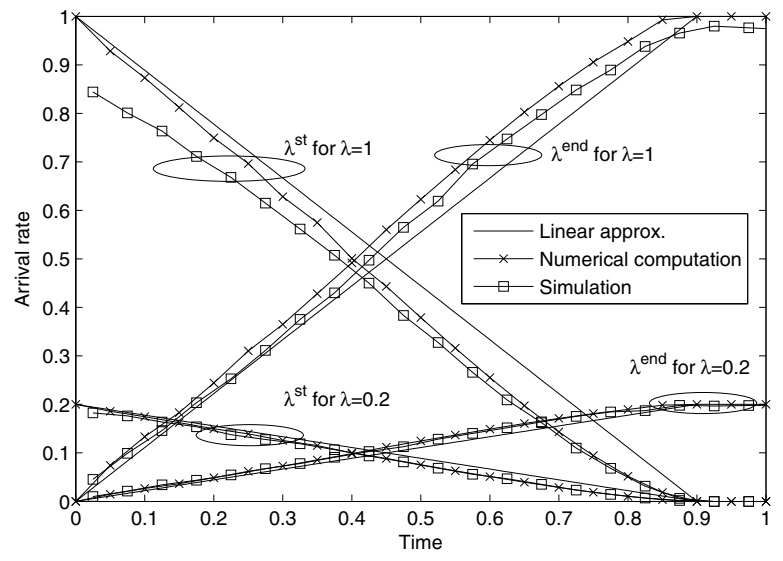

Fig. 3. Comparison of the linear approximation against the numerical solutions of (4) and (7) and simulation results $\lambda=0.2$ and $\lambda=1$ at $T=0.9$.

If a new transmission starts immediately after a previous transmission, some of the nodes in the network are already exposed to the previous transmission which results in a reduced arrival rate of colliding transmissions for the new transmission. This effect is not captured in our model which assumes consecutive transmissions are independent.

\section{B. Throughput}

Throughput of a CSMA network can be analyzed by inspecting the packet receptions at the base station. Fig. 4 demonstrates a series of packet receptions at the base station. The timeline consists of renewal intervals where each renewal interval corresponds to the time between the start of consecutive transmissions. The busy period of the renewal interval includes either a single successful transmission or a number of collided transmissions. If the busy period consists of a single transmission, its length is one. If a colliding transmission arrives after the start of the busy period, the length of the busy interval gets longer depending on the arrival time of the colliding transmission. The idle period in the renewal interval is the time required for a new transmission to arrive after the end of a busy period. Hence, the throughput of the CSMA network can be written as

$$
\sigma_{C S M A}^{T}=\frac{P_{s}}{E\left[t_{\text {busy }}\right]+E\left[t_{\text {idle }}\right]}
$$

where $t_{\text {busy }}$ and $t_{\text {idle }}$ denote the lengths of the busy and idle periods, respectively and $P_{s}$ represents the probability of success for the busy period, i.e., the probability that a single packet arrives in the busy period.

To derive the probability distribution of lengths of these periods, we refer to a branch of statistics called survival or reliability analysis [9]. The varying arrival rate of a colliding transmission is analogous to the hazard rate in this literature and probability of success is analogous to the survival probability up to the duration of a packet.

1) Probability of success: For a given hazard rate function, the probability of survival up to time $t$ is analogous to the probability of no collisions up to $t$, which is written as [9]:

$$
S^{s t}(t)=\exp \left\{-\int_{0}^{t} \lambda^{s t}(t) \mathrm{d} t\right\} .
$$

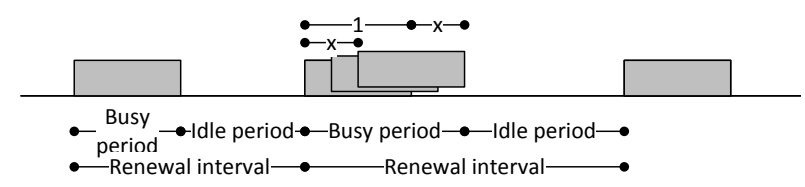

Fig. 4. Illustration of renewal intervals and busy periods. The length of the busy period is $1+X$ where $X$ is the arrival time of the last colliding transmission.

Using (8), the approximate probability of no collision during the vulnerable period can be obtained as follows:

$$
P_{s}=S^{s t}(T)=\exp \left\{-\int_{0}^{T} \lambda^{s t}(t) \mathrm{d} t\right\}=e^{-\frac{T}{2} \lambda} .
$$

2) Expected duration of the idle period: For a given survival function, the expectation of life can be obtained as [9]

$$
\mu=\int_{0}^{\infty} S(t) \mathrm{d} t
$$

which is analogous to the expected time till the arrival of a new transmission. Using (9), the survival function is given by

$$
\begin{aligned}
S^{e n d}(t) & =\exp \left\{-\int_{0}^{t} \lambda^{e n d}(\tau) \mathrm{d} \tau\right\} \\
& = \begin{cases}\exp \left\{-\frac{\lambda t^{2}}{2 T}\right\} & \text { if } t \leq T \\
\exp \left\{-\left(t-\frac{T}{2}\right) \lambda\right\} & \text { if } t>T\end{cases}
\end{aligned}
$$

Then, the mean duration of the idle period can be found as

$$
\begin{aligned}
E\left[t_{\text {idle }}\right] & =\int_{0}^{\infty} S^{\text {end }}(t) \mathrm{d} t \\
& =\sqrt{\frac{\pi T}{2 \lambda}} \operatorname{erf}\left(\sqrt{\frac{\lambda T}{2}}\right)+\frac{e^{-\frac{\lambda T}{2}}}{\lambda} .
\end{aligned}
$$

3) Expected duration of the busy period: Duration of the busy period is one for successful transmissions which occurs with probability $P_{s}$. If one or more colliding transmissions arrives in the vulnerable period, then the length of the busy period is $1+X$ where $X$ is the arrival time of the last colliding transmission as it can be seen from Fig. 4.

To obtain $E[X]$, we first find the probability of no arrivals occurring between $t$ and $T$.

$$
F_{X}(t)=\exp \left\{-\int_{t}^{T} \lambda^{s t}(\tau) \mathrm{d} \tau\right\}=e^{-\frac{\lambda t^{2}-2 \lambda t T+\lambda T^{2}}{2 T}}
$$

Then the expected value of $X$ can be found as:

$$
E[X]=\int_{0}^{T}\left(1-F_{X}(t)\right) \mathrm{d} t
$$

The expected length of the busy period is given by:

$$
\begin{aligned}
E\left[t_{\text {busy }}\right] & =1+E[X] \\
& =1+T-\sqrt{\frac{\pi T}{2 \lambda}} \operatorname{erf}\left(\sqrt{\frac{\lambda T}{2}}\right)
\end{aligned}
$$




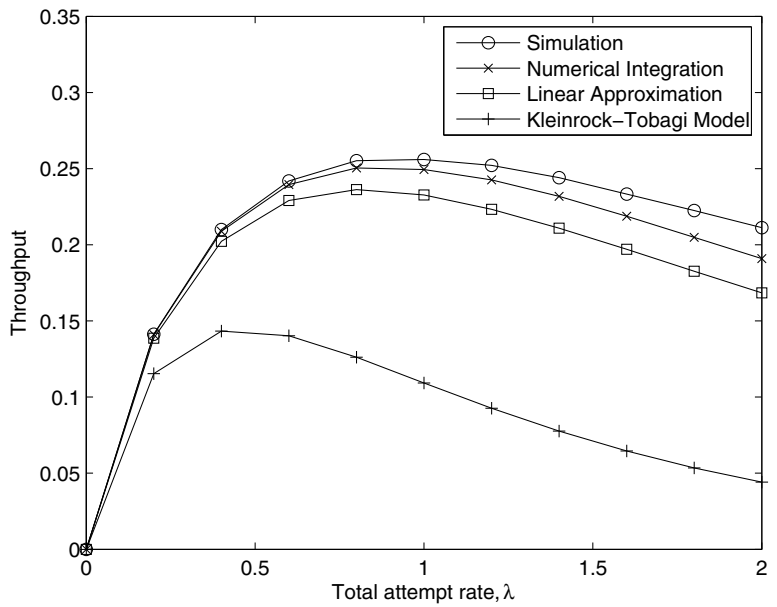

Fig. 5. Throughput as a function of attempt rate in the network for $T=1$.

4) Throughput of CSMA: Using (12), (15) and (18), we can obtain the throughput expression given by (10) as follows:

$$
\sigma_{C S M A}^{T}=\frac{\lambda e^{-\lambda \frac{T}{2}}}{\lambda(T+1)+e^{-\lambda \frac{T}{2}}}
$$

It should be noted that the proposed throughput function has the same form with the expression in [1] but the maximum delay is replaced by half of the maximum delay.

\section{NUMERICAL RESULTS}

In this section, we evaluate the proposed throughput analysis against simulation results. Fig. 5 depicts the throughput versus the total attempt rate in the network for $T=1$ and Fig. 6 plots the change in the maximum throughput (both in absolute value and percentage error with respect to simulations) as $T$ increases. Both figures include the throughput obtained by exact computation of $\lambda^{s t}$ and $\lambda^{\text {end }}$ by using numerical integration in (4) and (7), throughput expression obtained by the linear approximation as given by (19) and the throughput expression given in [1]. The discrepancy between the proposed models and the simulated throughput increases as the total attempt rate increases since this increase results in an increased dependency between consecutive transmissions as discussed in Sec. IV-A. The estimation errors for all three models also increase as $T$ increases. The numerical integration provides the closest result to the simulation as it estimates the maximum throughput with a $3 \%$ error. The linear approximation of the arrival rate function results in a $8 \%$ underestimation whereas the model given in [1] underestimates the maximum throughput with a $44 \%$ error.

\section{CONCLUSION}

We proposed an analysis of the throughput for a nonpersistent CSMA network in which we incorporate the spatiotemporal effects caused by large propagation delay by modeling the arrival rate of colliding transmissions after the start of a transmission and after the end of a transmission. We computed the throughput using an exact computation and with an approximate model which results in the simple throughput

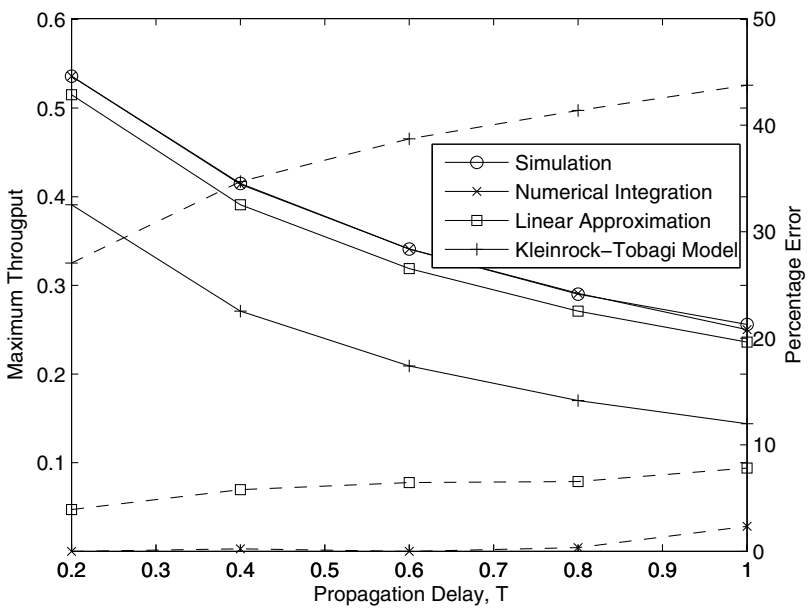

Fig. 6. The maximum throughput and the percentage error as $T$ increases. Dashed lines indicate the percentage error with respect to simulations.

expression (19). In estimating the maximum throughput, the model with numerical integration deviates from simulation results with $3 \%$ error and the linear approximation model results in an $8 \%$ error whereas the Kleinrock-Tobagi model results in an error of $44 \%$ when the maximum propagation delay equals to the packet transmission time. The simple expression given by (19) can be used for accurate estimation of throughput for CSMA networks in emerging applications with large propagation delays.

\section{ACKNOWLEDGMENT}

This work was supported by the European Commission in the framework of the FP7 Network of Excellence in Wireless Communications NEWCOM\#.

\section{REFERENCES}

[1] L. Kleinrock and F. Tobagi, "Packet switching in radio channelspart I: carrier sense multiple-access modes and their throughput-delay characteristics," IEEE Trans. Commun., vol. 23, no. 12, pp. 1400-1416, Dec. 1975.

[2] R. Rom and M. Sidi, "Multiple access protocols: performance and analysis," 1990.

[3] D. Chan, T. Berger, and L. Tong, "Carrier sense multiple access communications on multipacket reception channels: theory and applications to IEEE 802.11 wireless networks," IEEE Trans. Commun., vol. 61, no. 1, pp. 266-278, 2013.

[4] B. Nardelli and E. Knightly, "Closed-form throughput expressions for CSMA networks with collisions and hidden terminals," in Proc. 2012 IEEE INFOCOM, pp. 2309-2317.

[5] L. Dai, "Toward a coherent theory of CSMA and Aloha," IEEE Trans. Wireless Commun., vol. 12, no. 7, pp. 3428-3444, 2013.

[6] M. Koseoglu and E. Karasan, "Throughput modeling of single hop CSMA networks with non-negligible propagation delay," IEEE Trans. Commun., vol. 61, no. 7, pp. 2911-2923, 2013.

[7] R.-H. Gau, "Modeling the slotted nonpersistent CSMA protocol for wireless access networks with multiple packet reception," IEEE Commun. Lett., vol. 13, no. 10, pp. 797-799, 2009.

[8] Y. Zhu, Z. Jiang, Z. Peng, M. Zuba, J.-H. Cui, and H. Chen, "Toward practical MAC design for underwater acoustic networks," in Proc. 2013 IEEE INFOCOM, pp. 683-691.

[9] G. Rodríguez, "Lecture notes on generalized linear models," 2007. Available: http://data.princeton.edu/wws509/notes/. 